\title{
Rib Fracture Fixation Restores Inspiratory Volume and Peak Flow in a Full Thorax Human Cadaveric Breathing Model
}

\author{
Gerard P. Slobogean,' Hyunchul Kim, Joseph P. Russell, ${ }^{2}$ David J. Stockton, ${ }^{3}$ Adam H. Hsieh, \\ and Robert V. O'Toole ${ }^{1}$
}

${ }^{1}$ Department of Orthopaedics, University of Maryland School of Medicine, R. Adams Cowley Shock Trauma Center, Baltimore, Maryland, USA

${ }^{2}$ Fischell Department of Bioengineering, Orthopaedic Mechanobiology Laboratory, University of Maryland, College Park, Maryland, USA

${ }^{3}$ Department of Orthopaedics, University of British Columbia, Vancouver, British Columbia, Canada

*Corresponding author: Gerard P. Slobogean, Department of Orthopaedics, University of Maryland School of Medicine, R. Adams Cowley Shock Trauma Center, Baltimore, Maryland, USA. Tel: +1-4103286280, Fax:+1-4103282893, E-mail: gslobogean@umoa.umm.edu

Received 2015 April 2; Revised 2015 June 10; Accepted 2015 September 10.

\begin{abstract}
Background: Multiple rib fractures cause significant pain and potential for chest wall instability. Despite an emerging trend of surgical management of flail chest injuries, there are no studies examining the effect of rib fracture fixation on respiratory function.

Objectives:Using a novel full thorax human cadaveric breathing model, we sought to explore the effect of flail chest injury and subsequent rib fracture fixation on respiratory outcomes.

Patients and Methods: We used five fresh human cadavers to generate negative breathing models in the left thorax to mimic physiologic respiration. Inspiratory volumes and peak flows were measured using a flow meter for all three chest wall states: intact chest, left-sided flail chest (segmental fractures of ribs 3 - 7), and post-fracture open reduction and internal fixation (ORIF) of the chest wall with a precontoured rib specific plate fixation system.

Results: A wide variation in the mean inspiratory volumes and peak flows were measured between specimens; however, the effect of a flail chest wall and the subsequent internal fixation of the unstable rib fractures was consistent across all samples. Compared to the intact chest wall, the inspiratory volume decreased by $40 \pm 19 \%$ in the flail chest model $(\mathrm{P}=0.04)$. Open reduction and internal fixation of the flail chest returned the inspiratory volume to $130 \pm 71 \%$ of the intact chest volumes $(\mathrm{P}=0.68)$. A similar $35 \pm 19 \%$ decrease in peak flows was seen in the flail chest $(\mathrm{P}=0.007)$ and this returned to $125 \pm 71 \%$ of the intact chest following ORIF $(\mathrm{P}=0.62)$.

Conclusions: Negative pressure inspiration is significantly impaired by an unstable chest wall. Restoring mechanical stability of the fractured ribs improves respiratory outcomes similar to baseline values.
\end{abstract}

Keywords: Flail Chest, Inspiratory Volume, Operative Stabilization, Peak Flow, Rib Fixation

\section{Background}

The mechanics of respiration involve complex interactions between the lungs, chest wall, and changing intrathoracic pressure (1). A key static property of pulmonary mechanics is the outward elastic recoil of the chest wall that helps keep the lungs expanded and facilitates the diaphragm to generate the negative intra-thoracic pressure gradient responsible for inspiration $(1,2)$. Changes in the properties of the chest wall are likely to directly affect lung volumes and respiratory function (3).

Flail chest injuries represent significant chest wall trauma. With multiple segmental rib fractures the chest wall becomes grossly unstable, the thoracic volume decreases, and paradoxical inward motion of the flail segment is seen during inspiration. As a result, flail chest injuries are associated with prolonged ICU stays, ventilator associated complications, and long-term respiratory dysfunction (4-6).
Surgical fixation of flail chest injuries has been suggested to improve multiple critical care outcomes compared to traditional non-operative management $(7,8)$. While the benefits of surgical stabilization may seem intuitive, the mechanism for improved short-term clinical outcomes is unknown. Are critical care outcomes better because surgical stabilization minimizes pain from the constant movement of displaced fractures? Does fixation restore chest wall stability and allow proper negative-pressure inspiration? Or, is it a combination of both mechanisms that can facilitate earlier discontinuation of mechanical ventilation and avoidance of related complications?

Despite the potential patient benefits, operative stabilization has been described as a largely underutilized treatment for flail chest injuries $(4,9)$. As traumatologists' interest in rib fixation is renewed, an increase in rib specific implants and their laboratory testing has been

Copyright (C) 2015, Kashan University of Medical Sciences. This is an open-access article distributed under the terms of the Creative Commons Attribution-NonCommercial 4.0 International License (http://creativecommons.org/licenses/by-nc/4.0/) which permits copy and redistribute the material just in noncommercial usages, provided the original work is properly cited. 
observed (10-12). Biomechanical testing of rib fracture fixation is important to ensure new implants are appropriately designed, but also to help understand the influence of fixation on clinically important outcomes. To our knowledge, laboratory testing of rib fracture fixation has been limited to single-rib, load-to-failure biomechanical studies, with no laboratory studies exploring the effect of flail chest fixation on respiratory outcomes.

\section{Objectives}

The purpose of the current study was to: 1) create a full thorax cadaveric model of negative inspiration, 2) use the novel full thorax breathing model to create a large flail chest injury, and 3) explore the effect of rib fracture fixation on respiratory outcomes in this model. We hypothesized that our model would demonstrate the role of chest wall stability during negative pressure inspiration. Specifically, we hypothesized that a significant decrease in lung volume and peak inspiratory flow rate would be observed in the flail chest model, and that these respiratory outcomes would be improved by surgical fixation of the flail segment.

\section{Patients and Methods}

A full thorax negative pressure breathing model was developed to mimic physiologic breathing. Next, a fiverib flail chest segment was created to test the effect of surgical fixation on respiratory function. The primary outcomes of interest were inspiratory volume and peak inspiratory flow rate.

\subsection{Specimens}

Five fresh human thoraces were harvested within 4 days post mortem (mean 3 days, range 2 - 4 days). The mean age of the donors was 77 years (standard deviation, 6 years); 2 of the specimens were female (Table 1 ). The postmortem human subjects (PMHS) were obtained through the Maryland State Anatomy Board. All specimens were initially washed with a germicidal soap, disinfected with an arterial flush, and then stored at $4^{\circ}$ Celsius prior to experimental testing. On the morning of testing, the specimens were cross-sectioned at $10^{\text {th }}$ thoracic vertebra to provide access to the undersurface of the diaphragm. The remainder of the upper body was left intact.

\subsection{Breathing Model}

A negative pressure breathing model was created using the following steps: 1) The airway was accessed through a standard tracheostomy incision. A size 8 Shiley tracheostomy tube (inner diameter $7.6 \mathrm{~mm}$ ) was inserted into the trachea, and the cuff was inflated to seal the airway. 2) Caudal traction was applied to the diaphragm using a 5-pound weight attached to sutures in the inferior surface of the left hemi-diaphragm.3) A 40-French chest tube was inserted into the left thorax at the anterior axillary line between the $4^{\text {th }}$ and $5^{\text {th }}$ ribs. 4 ) To initiate inspiration, the chest tube was connected to a suction pump at 5 inches of mercury (inHg); this created negative intra-thoracic pressure and passive inflation of the left lung parenchyma. 5) To visually confirm lung inspiration and indirectly confirm an air tight system, a sealed "window" into the left thoracic cavity was created. The $3 \times 3 \mathrm{~cm}$ window was made between ribs 3 and 4 at the lateral sternal border, covered with clear plastic, and its perimeter sealed with an adhesive dressing (Ioban, 3M). A schematic diagram of the model design and testing setup is found in Figure 1.

\subsection{Flail Chest Model}

Respiratory outcomes were assessed in three sequential chest wall states: intact chest, flail chest, and post-open reduction and internal fixation (ORIF) chest. The intact chest had no fractures or disruption to the soft tissues, aside from the sealed window required for the breathing aspect of the model.

To access the chest wall and create a flail chest, anterior and posterior longitudinal incisions were made overlying ribs 3 - 7 along the anterolateral chest and posteromedial scapular border. Using an oscillating saw, two fractures per rib (ribs 3 - 7) were created while preserving the parietal pleura, intercostal muscles, and soft tissues within the flail segment and surrounding chest wall (Figure 2). The fracture sites were templated to ensure the rib-specific plates would span both segmental fractures of each rib with two screws of fixation on each end. Each fracture was carefully manipulated to ensure complete displacement from the intact ribs. The skin was then stapled closed and sealed with strips of Ioban dressing.

Open reduction and internal fixation of the flail chest was performed using rib-specific pre-contoured plates (MatrixRIB $^{\mathrm{TM}}$, DePuy Synthes). All five ribs (ribs 3 - 7) were stabilized with two screws at each side of a fracture site for a total of eight screws per rib. The skin incisions were again closed with staples and sealed with the adhesive dressing before testing of the post-ORIF chest wall.

\subsection{Respiratory Outcomes}

The inspiratory peak flow and total volume were measured in each chest wall state as described below. Pilot testing had demonstrated that peak flows occurred within the first few seconds of our model, with only modest volume increases as the inspiration was prolonged to 10 seconds. Furthermore, analysis of the preliminary specimens suggested a similar magnitude of results when using 2, 3, or 10 second inspirations. As a result, we chose a 2 Second inspiratory phase to approximate a respiratory rate of 12 breaths per minute and a 1: 2 inspiration to expiration ratio as frequently seen during normal breathing.

The respiratory outcomes were measured using a commercial flow sensor (D6F-50A-6000, Omron Electronic company, Schaumburg, IL) connected to the tracheostomy tube. Flow meter data was collected using a data ac- 
quisition card (DAQ 6008-USB card, National Instrument, Austin, TX) and processed using Labview v8.6. Integration of the flow rate calculated the total inspiratory volume. All parameters were measured 5 Times; the lowest and highest trials were discarded and a mean value for the remaining three trials was kept.

\subsection{Statistical Analysis}

Descriptive statistics were used for all outcomes of interest. The mean and standard deviation (SD) was used to describe all continuous data. Counts and proportions were used for non-continuous variables. A paired t-test was used to test for differences between the intact chest and the flail chest, the flail chest and post-ORIF chest, and the intact chest and post-ORIF chest results.

\section{Results}

A significant decrease in respiratory outcomes was observed in the flail chest compared to the intact chest wall model (Tables 2 and 3). A mean 40\% relative decrease in inspiratory volumes was observed in the unstable chest wall state compared to the intact chest wall $(\mathrm{P}=0.04)$. Similarly, a mean decrease of $3.3 \mathrm{~L} / \mathrm{min}$ was observed in the peak flow rates of the flail chest models compared to the intact chest wall $(\mathrm{P}=0.007)$.

Minimal variations in peak inspiratory volumes and flow rates were observed within the individual specimens; however, a substantial range of values were observed between donors. In the flail chest model, the mean inspiratory volume decreased to $40 \% \pm 19 \%$ of the intact chest values. In the post-ORIF model, the mean inspiratory volume increased to $130 \% \pm 71 \%$ of the intact chest values. The mean peak flow rate for the intact chest model was $5.2 \mathrm{~L} / \mathrm{min}(\mathrm{SD}, 2.2 \mathrm{~L} / \mathrm{min})$. In the flail chest model, the mean peak flow rate was $35 \% \pm 19 \%$ of the mean peak flow rate in the intact model. In post-ORIF model, the mean peak flow rate increased to $125 \% \pm 71 \%$ of the mean peak flow rate in the intact model (Table 3 ).

Once the segmental fractures of the flail chest were stabilized with ORIF, significant increases in the respiratory outcomes were observed (Tables 2 and 3). When comparing the respiratory outcomes between the flail chest and the post-ORIF chest wall, a 263\% increase in respiratory volume and a 300\% increase in peak flow was observed $(\mathrm{P}<$ $0.02)$.

The post-ORIF inspiratory volumes and flow rates were very similar to the initial intact chest wall values. No statistically significant differences were detected between the two chest wall states, although the mean estimates for the post-ORIF chest wall tended to be higher $(\mathrm{P}>0.68)$.

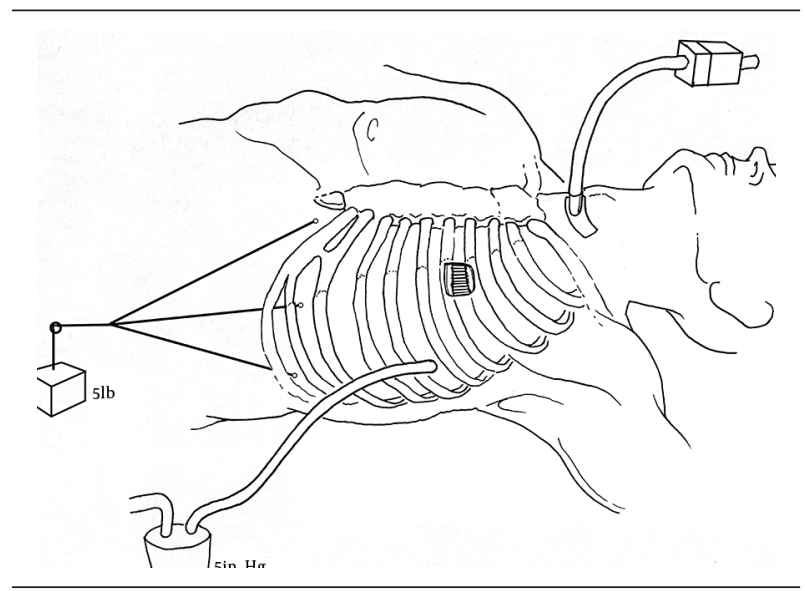

Figure 1. Model Design

\begin{tabular}{lccccc}
\hline Table 1. Specimen Characteristics & & & & \\
\hline Specimen & $\mathbf{1}$ & $\mathbf{2}$ & $\mathbf{3}$ & $\mathbf{4}$ & $\mathbf{5}$ \\
\hline Age, $\mathbf{y}$ & 77 & 68 & 83 & 78 & 80 \\
Gender & Male & Female & Male & Male & Female \\
Cause of Death & COPD & Bowel cancer & Myocardial infarction & Liver cancer & Myocardial infarction \\
Post-Mortem $(\mathbf{d})$ & 4 & 2 & 2 & 3 & 3 \\
\hline
\end{tabular}
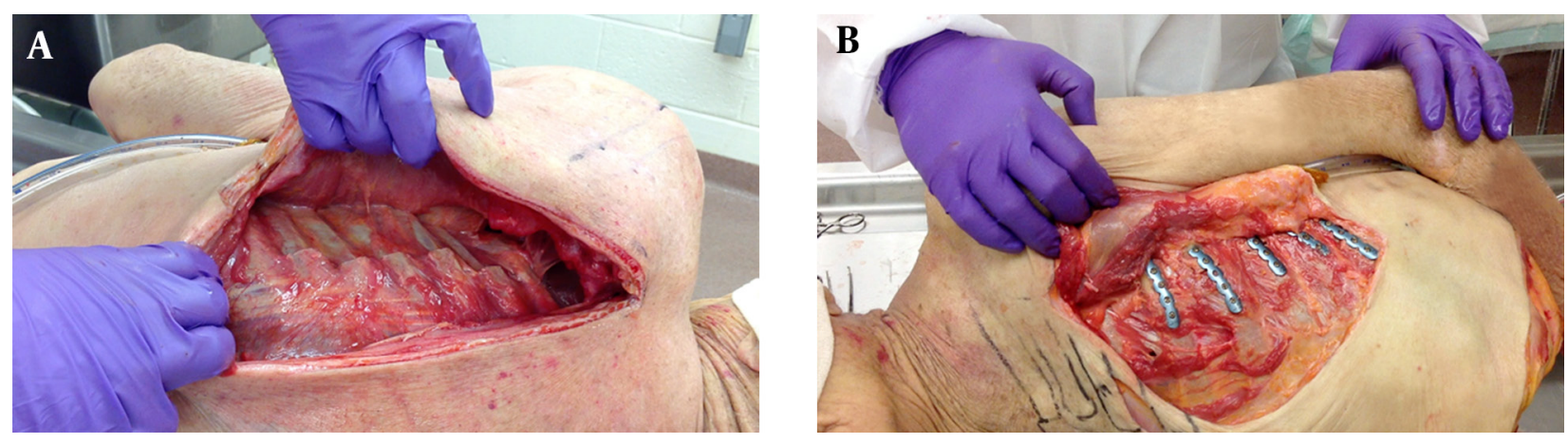

Figure 2. Fractures and fixation 
Slobogean GP et al.

\begin{tabular}{|c|c|c|c|c|c|c|}
\hline Specimen & $\mathbf{1}$ & 2 & 3 & 4 & 5 & Mean \pm SD \\
\hline \multicolumn{7}{|l|}{ Intact Chest } \\
\hline Volume $^{\mathrm{a}}$ & 22 & 38 & 57 & 104 & 145 & $73 \pm 50$ \\
\hline \multicolumn{7}{|l|}{ Flail Chest } \\
\hline Volume $^{\mathrm{a}}$ & 8 & 14 & 33 & 13 & 84 & $30 \pm 32^{b}$ \\
\hline$\%$ of Intact & 35 & 37 & 58 & 12 & 58 & $40 \pm 19$ \\
\hline \multicolumn{7}{|c|}{ Post-ORIF Chest } \\
\hline Volume $^{\mathrm{a}}$ & 32 & 95 & 45 & 91 & 133 & $79 \pm 41^{\mathrm{c}, \mathrm{d}}$ \\
\hline$\%$ of Intact & 143 & 247 & 78 & 88 & 92 & $130 \pm 71$ \\
\hline \multicolumn{7}{|c|}{$\begin{array}{l}\mathrm{a}_{\text {Volume are presented as percent. }} \\
\mathrm{b}_{\text {Comparison of intact chest and flail chest volumes, } \mathrm{P}=0.04 \text {. }} \\
\mathrm{c}_{\mathrm{C}} \text { Comparison of flail chest and post-ORIF chest volumes, } \mathrm{P}=0.02 \text {. } \\
\mathrm{d}_{\text {Comparison of intact chest and post-ORIF chest volumes, } \mathrm{P}=0.68 \text {. }} \text {. }\end{array}$} \\
\hline \multicolumn{7}{|c|}{ Table 3. Mean Peak Inspiratory Flow Rates } \\
\hline Specimen & $\mathbf{1}$ & 2 & 3 & 4 & 5 & Mean \pm SD \\
\hline \multicolumn{7}{|l|}{ Intact Chest } \\
\hline Flow $^{\mathrm{a}}$ & 3.4 & 2.8 & 5.6 & 5.9 & 8.2 & $5.2 \pm 2.2$ \\
\hline \multicolumn{7}{|l|}{ Flail Chest } \\
\hline Flow $^{\mathrm{a}}$ & 0.60 & 1.4 & 2.7 & 0.75 & 3.9 & $1.9 \pm 1.4^{b}$ \\
\hline$\%$ of Intact & 17 & 50 & 49 & 13 & 47 & $35 \pm 19$ \\
\hline \multicolumn{7}{|c|}{ Post-ORIF Chest } \\
\hline Flow $^{\mathrm{a}}$ & 3.8 & 7.0 & 3.9 & 5.1 & 8.8 & $5.7 \pm 2.1^{\mathrm{c}, \mathrm{d}}$ \\
\hline$\%$ of Intact & 111 & 249 & 69 & 87 & 107 & $125 \pm 71$ \\
\hline
\end{tabular}

\section{Discussion}

The results of our study demonstrate significant decreases in inspiratory volumes and peak flow rates with a flail chest injury, and restoration of respiratory outcomes to pre-injury values with surgical fixation. These results were obtained in a novel model using fresh, full-thorax post-mortem specimens and a negative pressure breathing mechanism to better simulate physiological respiratory mechanics. These data confirm our hypothesis that negative pressure inspiration requires chest wall stability, and that rib fracture fixation appears to restore functional stability.

The results of our model are consistent with previous clinical reports suggesting improved respiratory outcomes in flail chest patients treated with surgical fixation. In a randomized controlled trial of wire fixation versus non-operative treatment, Granetzny et al reported better pulmonary function test (PFT) results at twomonths post-injury in the operative group (4). Bottlang et al. prospectively evaluated 20 patients with flail chest injury fixed with MatrixRIB implants and found that at three months, patients had regained $84 \%$ of their ex- pected forced vital capacity (13). Similarly, Lardinois et al prospectively evaluated PFTs in 50 patients with flail chest injuries treated with plate fixation (14). Based on their results, the authors concluded that surgical fixation minimizes a post-injury restrictive lung pattern, with only $10 \%$ of their subjects experiencing a total lung capacity less than $85 \%$ of the predicted value. One randomized trial by Marasco et al. failed to show a difference in spirometry outcomes at three months, however this study used absorbable stabilization (Inion OTPSTM, Inion Inc.), not rigid fixation (15). Our results extend the work of these previous clinical studies by demonstrating a purely mechanical association between chest wall stability and negative pressure inspiratory volumes and peak flows.

The current study also extends previous basic science investigations of rib fixation implants by utilizing a novel testing model. Prior authors have often used a single rib loaded in compression to test the biomechanical properties of implants $(10,12)$. Although such models provide important information about the implant's performance 
in an isolated rib fracture, this is an obvious limitation to understanding the effects of the implant in the context of the entire chest wall. The soft tissues within the flail segment and the surrounding intact ribs must also be considered. Furthermore, the ability of the current model to measure changes in respiratory parameters between the intact, flail, and post-ORIF chest wall highlights the clinical relevance of our model.

Several other design factors of our negative intra-thoracic pressure breathing model require further discussion. We sought to design a whole chest model of a severe flail chest injury because we felt single rib models were too simplistic and failed to provide the clinically-relevant link to respiratory function that is needed to provide a biomechanical rationale for rib fixation. Moreover, negative intra-thoracic pressure respirations were felt to be a necessary design parameter since chest wall instability behaves quite differently during positive-pressure ventilation. Early versions of the model did not have diaphragm traction and permitted paradoxical cephalad movement of the diaphragm and decrease of the intrathoracic volume which is opposite to normal physiology. The addition of a chest tube on suction and a weight attached to the diaphragm helped artificially replicate negative pressure in vivo conditions and added to the face validity of our model. Regardless, the general observations of worse respiratory outcomes in the flail chest model and subsequent restoration of respiratory function after fixation in all subjects, suggest that these potential limitations do not alter the conclusions of the study.

Despite demonstrating convincing changes in respiratory outcomes within the model, the results of our study must be interpreted within the context of several parameters. The study's sample size involved five specimens. Although this is a relatively small number, it was appropriately powered to detect differences between the modeled injury states because of the large effect sizes observed and the statistical power gained by using repeated measures within a specimen (paired t-tests). It should also be noted that some study subjects had pre-existing obstructive lung disease and all of the subjects were elderly. Future research may benefit from analyzing a series of younger patients with normal lungs, although cadaver specimen access to a younger population is often challenging. In order to maximize the differences between chest wall states, we chose to model a very large flail chest injury involving five "pillar" ribs and the longest segmental fragments that would still facilitate a single plate to span both fractures per rib. It is likely that smaller flail segments will have less pronounced respiratory effects. Furthermore, all fractures within the flail chest were surgically stabilized. This required an extensive extra-thoracic dissection of the parascapular muscles that many surgeons may wish to avoid, particularly since it has been suggested that is unnecessary to stabilize both segmental fractures of all fractured ribs in a flail chest $(16,17)$. Finally, this breathing model is able to successfully replicate a negative intra-thoracic pres- sure respiratory mechanism; however, the effect of the intercostal muscles was not considered. Since most flail chest injuries are accompanied by damage to the intercostal muscles and previous animal models of flail chest have demonstrated that paradoxical chest wall motion is primarily driven by the changes in intra-thoracic pressure (18), we do not believe this limitation in our model should significantly alter our conclusions.

As surgical fixation of rib fractures and flail chest injuries continues to gain clinical popularity, several important questions remain. The timing of fixation, the role of pulmonary contusion as a contraindication, and the number of rib fractures necessary to stabilize in order to experience a benefit are unknown. While prospective clinical studies are necessary to definitively answer these questions, further biomechanical studies will greatly improve our understanding of how to manage these fractures. For example, stabilizing multiple segmental rib fractures may detrimentally over-stiffen the entire chest wall. The biomechanical effect of sequentially adding additional fixation on the chest wall stiffness is a potential area of investigation; this is particularly relevant since some authors have suggested that stiffness mismatch between the implants and native ribs is a cause of screw loosening (19).

The results of our study provide basic science data to support the assertion that rib fixation confers a mechanical benefit to the respiration of patients with flail chest injuries. This study also outlines a negative-pressure breathing model that mimics physiologic inspiration and allows simultaneous testing of rib fracture fixation. As open reduction and internal fixation of fib fractures continues to grow in popularity greater clinical and biomechanical understanding of this intervention is required. The current study provides clinically relevant, basic science data to demonstrate an association between chest wall stability and inspiratory outcomes in a negative pressure breathing model of flail chest injuries.

\section{Acknowledgments}

The authors would like to thank Mr. Ronald Wade, Director of the Anatomical Services Division, the Maryland State Anatomy Board as well as Mr. Dan Hensley and Mr. Tony Pleasant from the Maryland State Anatomy Board for their assistance in preparation of the specimens and laboratory support. The authors would also like to recognize the artistic contributions of Mr. Mark Wieber.

\section{Footnotes}

Authors' Contribution:The following authors made contributions to study conception and design (Gerard P. Slobogean, Hyunchul Kim, Adam H. Hsieh, Robert V. O'Toole); acquisition, analysis, and interpretation of data (Gerard P. Slobogean, Hyunchul Kim, Adam H. Hsieh, Joseph P. Russell, Robert V. O'Toole); drafting the manuscript (Gerard P. Slobogean, David J. Stockton, Robert 
V. O'Toole); and critical revisions (Gerard P. Slobogean, Hyunchul Kim, Adam H. Hsieh, Joseph P. Russell, David J. Stockton, and Robert V. O’Toole).

Funding/Support:The implants used in this study were donated by De Puy Synthes.

\section{References}

1. Widmaier EP, Raff H, Strang KT. Respiratory Physiology. In: Vander's Human Physiology: The Mechanisms of Body Function. 12 ed. New York: McGraw-Hill; 2011.

2. Boron WF. Mechanics of Ventilation. In: Medical Physiology: A Cellular and Molecular Approach. . 2 ed. Philadelphia: Saunders Elsevier; 2009.. pp. 630-51

3. Rhoades RA. Ventilation and the Mechanics of Breathing. In: Medical Physiology: Principles for Clinical Medicine. 4 ed. Baltimore: Lippincott Williams \& Wilkins; 2013.

4. Granetzny A, Abd El-Aal M, Emam E, Shalaby A, Boseila A. Surgical versus conservative treatment of flail chest. Evaluation of the pulmonary status. Interact Cardiovasc Thorac Surg. 2005;4(6):5837. doi:10.1510/icvts.2005.111807. [PubMed:17670487]

5. Mayberry J. Early stabilization of flail chest with locked plate fixation. J Orthop Trauma. 2011;25(11):648. doi: 10.1097| BOT.0b013e31822a542d. [PubMed: 22008859]

6. Tanaka H, Yukioka T, Yamaguti Y, Shimizu S, Goto H, Matsuda H, et al. Surgical stabilization of internal pneumatic stabilization? A prospective randomized study of management of severe flail chest patients. J Trauma. 2002;52(4):727-32. [PubMed:11956391]

7. de Jong MB, Kokke MC, Hietbrink F, Leenen LP. Surgical Management of Rib Fractures: Strategies and Literature Review. Scand J Surg. 2014;103(2):120-5. doi: 10.1177/1457496914531928. [PubMed: 24782038]

8. Slobogean GP, MacPherson CA, Sun T, Pelletier ME, Hameed SM. Surgical fixation vs nonoperative management of flail chest: a meta-analysis. J Am Coll Surg. 2013;216(2):302-11 e1. doi: 10.1016/j. jamcollsurg.2012.10.010. [PubMed: 23219148]

9. Dehghan N, de Mestral C, McKee MD, Schemitsch EH, Nathens A. Flail chest injuries: a review of outcomes and treatment practices from the
National Trauma Data Bank. J Trauma Acute Care Surg. 2014;76(2):4628. doi:10.1097/TA.0000000000000086. [PubMed:24458051]

10. Bottlang M, Helzel I, Long WB, Madey S. Anatomically contoured plates for fixation of rib fractures. J Trauma. 2010;68(3):611-5. doi: 10.1097/TA.ob013e3181a5b260. [PubMed:19935113]

11. Campbell N, Conaglen P, Martin K, Antippa P. Surgical stabilization of rib fractures using Inion OTPS wraps-techniques and quality of life follow-up. J Trauma. 2009;67(3):596-601. doi: 10.1097/TA.ob013e3181ad8cb7. [PubMed:19741406]

12. Sales JR, Ellis TJ, Gillard J, Liu Q, Chen JC, Ham B, et al. Biomechanical testing of a novel, minimally invasive rib fracture plating system. J Trauma. 2008;64(5):1270-4. doi: 10.1097| TA.ob013e31804a7fd5. [PubMed:18469649]

13. Bottlang M, Long WB, Phelan D, Fielder D, Madey SM. Surgical stabilization of flail chest injuries with MatrixRIB implants: a prospective observational study. Injury. 2013;44(2):232-8. doi: 10.1016/j.injury.2012.08.011. [PubMed: 22910817]

14. Lardinois D, Krueger T, Dusmet M, Ghisletta N, Gugger M, Ris HB Pulmonary function testing after operative stabilisation of the chest wall for flail chest. Eur J Cardiothorac Surg. 2001;20(3):496501. [PubMed: 11509269]

15. Marasco SF, Davies AR, Cooper J, Varma D, Bennett V, Nevill R, et al. Prospective randomized controlled trial of operative rib fixation in traumatic flail chest. J Am Coll Surg. 2013;216(5):924-32. doi:10.1016/j.jamcollsurg.2012.12.024. [PubMed: 23415550]

16. Marasco S, Liew S, Edwards E, Varma D, Summerhayes R. Analysis of bone healing in flail chest injury: do we need to fix both fractures per rib? J Trauma Acute Care Surg. 2014;77(3):452-8. doi: 10.1097/TA.0000000000000375. [PubMed: 25159250]

17. Voggenreiter G, Neudeck F, Aufmkolk M, Obertacke U, SchmitNeuerburg KP. Operative chest wall stabilization in flail chestoutcomes of patients with or without pulmonary contusion. $J$ Am Coll Surg. 1998;187(2):130-8. [PubMed: 9704957]

18. Cappello M, Legrand A, De Troyer A. Determinants of rib motion in flail chest. Am J Respir Crit Care Med. 1999;159(3):886-91. doi: 10.1164/ajrccm.159.3.9807089. [PubMed:10051267]

19. Engel C, Krieg JC, Madey SM, Long WB, Bottlang M. Operative chest wall fixation with osteosynthesis plates. $J$ Trauma. 2005;58(1):181-6. [PubMed:15674171] 